\title{
DISCUSSION
}

\section{Determination of critical slip surface in slope analysis}

\author{
S. K. SARMA and D. TAN (2006). Géotechnique 56, No. 8, 539-550
}

\section{J.-J. Wang and $\mathbf{X}$. Lin, Chongqing Jiaotong University, $P$. R. China}

The authors have developed a new method for determining the critical slip surface in slope stability analysis based on the limit equilibrium technique. In the new method, six unknowns - the angles $\alpha_{i}$ and $\delta_{i+1}$, the forces on the slip surface $N_{i}^{\prime}$ and $T_{i}$, and the forces on the interslice boundary $E_{i+1}^{\prime}$ and $X_{i+1}$ - can be obtained from the six equations (1), (2), (3), (4), (6) and (9). It is noted that the angle $\delta_{i+1}$ made by the interslice boundary with the vertical is also an unknown. In this way, it is inconvenient to solve the problem of the slope stability. Another more convenient method for determining the critical slip surface in slope analysis is developed in this discussion.

For a simpler homogeneous soil slope, the angle $\delta_{i+1}$ can approximately be obtained by Rankine's theory (Rankine, 1857) as

$$
\delta_{i+1}=\frac{\pi}{4}-\frac{\bar{\phi}_{i+1}^{\prime}}{2}
$$

In order to obtain the factor of safety of the analysed slope conveniently, the factor of safety on the slip surface is defined as (Bishop, 1955)

$$
F_{\mathrm{s}}=\frac{N_{i}^{\prime} \tan \phi_{i}^{\prime}+c_{i}^{\prime} b_{i} \sec \alpha_{i}}{T_{i}}
$$

The forces acting on the inclined slice are shown in Fig. 14. The equilibrium conditions in the horizontal and vertical directions for the forces acting on the slice are respectively expressed by

$$
\begin{aligned}
& -N_{i}^{\prime} \sin \alpha_{i}-U_{i} \sin \alpha_{i}+T_{i} \cos \alpha_{i} \\
& +E_{i}^{\prime} \sin \left(90-\delta_{i}-\bar{\phi}_{i}^{\prime}\right)+P W_{i} \cos \delta_{i}-X_{i}^{\prime} \sin \delta_{i} \\
& -E_{i+1}^{\prime} \sin \left(90-\delta_{i+1}-\bar{\phi}_{i+1}^{\prime}\right)-P W_{i+1} \cos \delta_{i+1} \\
& \quad+X_{i+1}^{\prime} \sin \delta_{i+1}-Q_{i}-H_{i}=0 \\
& N_{i}^{\prime} \cos \alpha_{i}+U_{i} \cos \alpha_{i}+T_{i} \sin \alpha_{i} \\
& \quad-E_{i}^{\prime} \cos \left(90-\delta_{i}-\bar{\phi}_{i}^{\prime}\right)-P W_{i} \sin \delta_{i}-X_{i}^{\prime} \cos \delta_{i} \\
& \quad+E_{i+1}^{\prime} \cos \left(90-\delta_{i+1}-\bar{\phi}_{i+1}^{\prime}\right)+P W_{i+1} \sin \delta_{i+1} \\
& +X_{i+1}^{\prime} \cos \delta_{i+1}-W_{i}-V_{i}=0
\end{aligned}
$$

where $Q_{i}$ is the total horizontal inertia force induced by earthquakes under seismic conditions; $H_{i}$ and $V_{i}$ are the horizontal and vertical forces acting on the upper surface of the slice, respectively; and $X_{i+1}^{\prime}=c_{i+1}^{\prime} d_{i+1}$.

The equilibrium condition for the moments of all the forces on the slice about the corner point A (Fig. 14) is given by

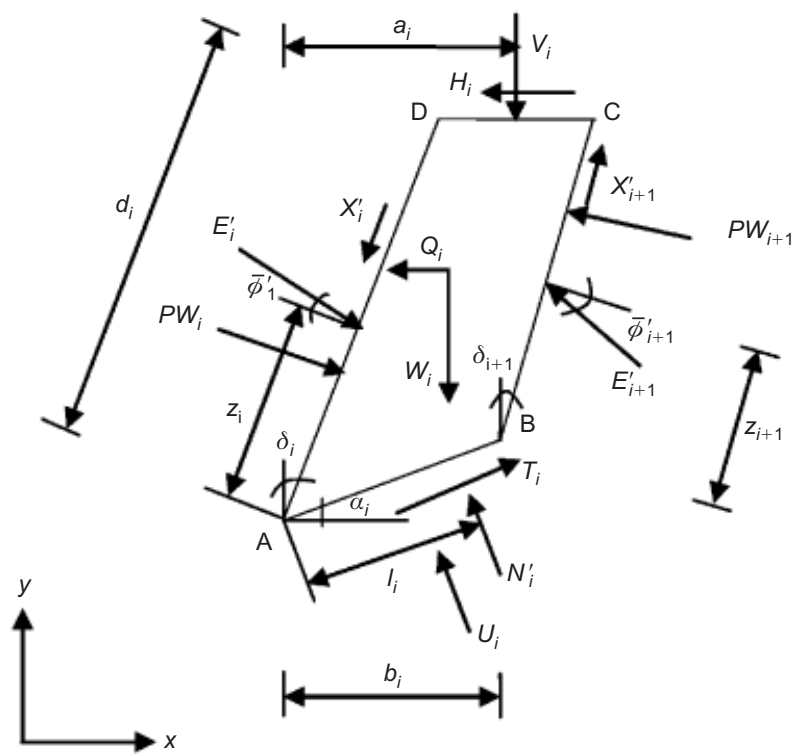

Fig. 14. Forces acting on an inclined slice

$$
\begin{aligned}
N_{i}^{\prime} l_{i} & +U_{i} l_{i}^{\prime}+X_{i+1}^{\prime} b_{i} \sec \alpha_{i} \sin \left(90-\alpha_{i}-\delta_{i+1}\right) \\
& +E_{i+1}^{\prime} \sin \bar{\phi}_{i+1}^{\prime} b_{i} \sec \alpha_{i} \sin \left(90-\alpha_{i}-\delta_{i+1}\right) \\
& +E_{i+1}^{\prime} \cos \bar{\phi}_{i+1}^{\prime}\left[z_{i+1}+b_{i} \sec \alpha_{i} \cos \left(90-\alpha_{i}-\delta_{i+1}\right)\right] \\
& +P W_{i+1}\left[z_{i+1}^{\prime}+b_{i} \sec \alpha_{i} \cos \left(90-\alpha_{i}-\delta_{i+1}\right)\right] \\
& +H_{i} d_{i} \cos \delta_{i}+Q_{i} y_{i}-W_{i} x_{i}-V_{i} a_{i}-P W_{i} z_{i}^{\prime} \\
& -E_{i}^{\prime} \cos \bar{\phi}_{i}^{\prime} z_{i}=0
\end{aligned}
$$

where $l_{i}^{\prime}$ and $z_{i}^{\prime}$ are the distances from the acting points of the forces $U_{i}$ and $P W_{i}$ to the point A, respectively; $z_{i+1}^{\prime}$ is the distance from the acting point of the force $P W_{i+1}$ to the point $\mathrm{B} ; x_{i}$ and $y_{i}$ are the horizontal and vertical distances from the centre of gravity of the slice to the point A, respectively; and $a_{i}$ is the horizontal distance from the acting point of the force $V_{i}$ to the point A. All of the six distances are known for any slice of an analysed slope.

When assuming that the normal stress distribution in any of the slip surface $\mathrm{AB}$, and interslice boundaries $\mathrm{AD}$ and $\mathrm{BC}$, is linear, the total effective normal stresses acting on the slip surface and interslice boundary can be rewritten as

$$
\begin{aligned}
& N_{i}^{\prime}=\frac{1}{2}\left(\sigma_{i}^{\prime}+\sigma_{i+1}^{\prime}\right) b_{i} \sec \alpha_{i} \\
& E_{i}^{\prime}=\frac{1}{2}\left(\sigma_{i}^{\prime 0}+\sigma_{i}^{\prime}\right) d_{i}
\end{aligned}
$$

The distances $l_{i}$ and $z_{i}$ from the acting points of the effective forces $N_{i}^{\prime}$ and $E_{i}^{\prime}$ to the point $\mathrm{A}$ are given by 


$$
\begin{aligned}
& l_{i}=\frac{b_{i} \sec \alpha_{i}}{3} \cdot \frac{\sigma_{i}^{\prime}+2 \sigma_{i+1}^{\prime}}{\sigma_{i}^{\prime}+\sigma_{i+1}^{\prime}} \\
& z_{i}=\frac{d_{i}}{3} \cdot \frac{2 \sigma_{i}^{\prime 0}+\sigma_{i}^{\prime}}{\sigma_{i}^{\prime 0}+\sigma_{i}^{\prime}}
\end{aligned}
$$

Substituting equations (42) to (45) into equations (39) to (41), and considering equation (38), yields the following equations:

$$
\begin{aligned}
& \frac{1}{2}\left(\sigma_{i}^{\prime}+\sigma_{i+1}^{\prime}\right) b_{i}\left(\frac{\tan \phi_{i}^{\prime}}{F_{\mathrm{s}}}-\tan \alpha_{i}\right) \\
& +\frac{1}{2}\left({\sigma_{i}^{\prime}}^{0}+\sigma_{i}^{\prime}\right) d_{i} \sin \left(90-\delta_{i}-\bar{\phi}_{i}^{\prime}\right) \\
& -\frac{1}{2}\left(\sigma_{i+1}^{\prime 0}+\sigma_{i+1}^{\prime}\right) d_{i+1} \sin \left(90-\delta_{i+1}-\bar{\phi}_{i+1}^{\prime}\right) \\
& +\frac{c_{i}^{\prime} b_{i}}{F_{\mathrm{s}}}-\bar{c}_{i}^{\prime} d_{i} \sin \delta_{i}+\bar{c}_{i+1}^{\prime} d_{i+1} \sin \delta_{i+1} \\
& -U_{i} \sin \alpha_{i}+P W_{i} \cos \delta_{i}-P W_{i+1} \cos \delta_{i+1} \\
& -Q_{i}-H_{i}=0 \\
& \frac{1}{2}\left(\sigma_{i}^{\prime}+\sigma_{i+1}^{\prime}\right) b_{i}\left(1+\frac{\tan \phi_{i}^{\prime} \tan \alpha_{i}}{F_{\mathrm{s}}}\right) \\
& -\frac{1}{2}\left(\sigma_{i}^{\prime 0}+\sigma_{i}^{\prime}\right) d_{i} \cos \left(90-\delta_{i}-\bar{\phi}_{i}^{\prime}\right) \\
& +\frac{1}{2}\left(\sigma_{i+1}^{\prime 0}+\sigma_{i+1}^{\prime}\right) d_{i+1} \cos \left(90-\delta_{i+1}-\bar{\phi}_{i+1}^{\prime}\right) \\
& +\frac{c_{i}^{\prime} b_{i} \tan \alpha_{i}}{F_{\mathrm{s}}}-\bar{c}_{i}^{\prime} d_{i} \cos \delta_{i}+\bar{c}_{i+1}^{\prime} d_{i+1} \cos \delta_{i+1} \\
& +U_{i} \cos \alpha_{i}-P W_{i} \sin \delta_{i}+P W_{i+1} \sin \delta_{i+1} \\
& -W_{i}-V_{i}=0 \\
& \frac{1}{6} b_{i}^{2}\left(\sigma_{i}^{\prime}+2 \sigma_{i+1}^{\prime}\right) \sec ^{2} \alpha_{i}-\frac{1}{6} d_{i}^{2}\left(2 \sigma_{i}^{\prime 0}+\sigma_{i}^{\prime}\right) \cos \bar{\phi}_{i}^{\prime} \\
& +\frac{1}{2} d_{i+1} b_{i}\left(\sigma_{i+1}^{\prime 0}+\sigma_{i+1}^{\prime}\right) \sec \alpha_{i} \\
& \times \cos \left(90-\alpha_{i}-\delta_{i+1}-\bar{\phi}_{i+1}^{\prime}\right) \\
& +\frac{1}{6} d_{i+1}^{2} \cos \bar{\phi}_{i+1}^{\prime}\left(2 \sigma_{i+1}^{\prime 0}+\sigma_{i+1}^{\prime}\right) \\
& +\bar{c}_{i+1}^{\prime} d_{i+1} b_{i} \sec \alpha_{i} \sin \left(90-\alpha_{i}-\delta_{i+1}\right) \\
& +U_{i} l_{i}^{\prime}-P W_{i} z_{i}^{\prime} \\
& +P W_{i+1}\left[z_{i+1}^{\prime}+b_{i} \sec \alpha_{i} \cos \left(90-\alpha_{i}-\delta_{i+1}\right)\right] \\
& +H_{i} d_{i} \cos \delta_{i}+Q_{i} y_{i}-W_{i} x_{i}-V_{i} a_{i}=0
\end{aligned}
$$

From equations (46)-(48) it is clear that, for the $i$ th slice, four unknowns need to be solved: $F_{\mathrm{s}}, \sigma_{i+1}^{\prime}, \sigma_{i+1}^{\prime 0}$ and $\alpha_{i}$. For the whole slope the number of all unknowns is $(3 n-1)$ one for $F_{s},(n-1)$ for $\sigma_{i+1}^{\prime},(n-1)$ for $\sigma_{i+1}^{\prime 0}$ and $n$ for $\alpha_{i}$ and the number of all equations is $3 n$, so that the problem can be solved.

For the first slice (Fig. 1) the effective normal stress at the starting point of the slip surface is obtained from the Mohr circle. If there is no surcharge in the starting point, the effective normal stress is given by

$$
\sigma_{1}^{\prime}=\frac{c_{1}^{\prime} \cos \phi_{1}^{\prime}}{F_{\mathrm{s}}}
$$

Considering a value for the factor of safety $F_{\mathrm{s}}$, all other unknowns $\sigma_{2}^{\prime}, \sigma_{2}^{\prime 0}$ and $\alpha_{1}$ can be obtained. Then, for the $i$ th slice from 2 and $(n-1)$, the unknowns can also be obtained using the given value of $F_{\mathrm{s}}$. For the last slice the effective normal stress $\sigma_{n+1}^{\prime 0}$ at the ending point of the slip surface is also obtained from the Mohr circle. If there is no surcharge in the ending point, the effective normal stress is given by

$$
\sigma_{n+1}^{\prime 0}=\frac{c_{n}^{\prime} \cos \phi_{n}^{\prime}}{F_{\mathrm{s}}}
$$

Hence the unknowns of the last slice are only $F_{\mathrm{s}}, \sigma_{n+1}^{\prime}$ and $\alpha_{n}$. A new value for the factor of safety $F_{\mathrm{s}}$ can be obtained from equations (46)-(48) for the last slice by comparing the calculated value for the factor of safety with the value given above, considering another new value for the factor of safety, and repeating the computation until the problem is solved. The critical slip surface and critical factor of safety are also obtained.

\section{Authors' reply}

The authors would like to thank the discussers for their interest in our work and their discussion of our paper. It is clear that the limit equilibrium technique of slope stability analysis can be modified in different ways to obtain solutions. Having gone through the discussion material, we would like to make the following comments on the discussers' note.

We know that, given $\alpha_{i}$ and $\delta_{i+1}$, the factor of safety/ critical acceleration can be determined as in the Sarma (1979) method. In the discussers' method $\alpha_{i}$ is an unknown and $\delta_{i+1}$ is a function of $\alpha_{i}$ for each slice, and therefore they need one extra equation for each slice compared with the traditional methods. It seems that they adjust the angle $\alpha_{i}$ so that the resulting normal stresses match with the linear stress distribution both along the slip surface and along the interslice boundaries. Therefore they will get a solution, but in the absence of numerical examples it is not clear whether the solution will be acceptable. Also, it is not clear what friction angle they are going to use for defining the interslice angle when either the factor of safety is unknown or/and when the slope is non-homogeneous.

Their definition of $E_{i+1}^{\prime}$ is not appropriate. If $E_{i+1}^{\prime}$ represents the inclined force, then $X_{i+1}$ is a component of $E_{i+1}^{\prime}$ and should not appear in their equation or in their Fig. 14. If $E_{i+1}^{\prime}$ represents the normal force acting on the interslice boundary, then it should not be inclined.

The discussers' assumption that $X_{i+1}=c_{i+1} d_{i+1}+$ $E_{i+1}^{\prime} \tan \phi_{i+1}$ is not proper. Because the discussers are doing a factor of safety analysis, the interslice boundary should not be in limiting equilibrium (i.e. a factor of safety of unity). We believe that the factor of safety in interslice boundaries should be greater than or equal to the factor of safety on the slip surface, $F_{\mathrm{s}}$, as discussed by Janbu (1973).

The more severe deficit of the discussers' work is that, for a given slip surface and interslice boundaries, their method cannot give a factor of safety or critical acceleration. Because the calculation of factor of safety is dependent on $\sigma_{i}^{\prime}$ and $\sigma_{i+1}^{\prime 0}$, for a given slip surface and interslice boundaries it can give an infinite number of factors of safety.

Unfortunately, the discussers do not provide any numerical examples to prove their method; furthermore, they do not discuss non-homogeneous cases. It would have been interesting to see some results compared with ours.

\section{REFERENCE}

Rankine, W. J. M. (1857). On the stability of loose earth. Phil. Trans. R. Soc. London 147, 9-27. 